Existence results for first order boundary value problems for fractional differential equations with four-point integral boundary conditions

Mohamed Abdalla Darwish and Sotiris K. Ntouyas 


\title{
EXISTENCE RESULTS FOR FIRST ORDER BOUNDARY VALUE PROBLEMS FOR FRACTIONAL DIFFERENTIAL EQUATIONS WITH FOUR-POINT INTEGRAL BOUNDARY CONDITIONS
}

\author{
MOHAMED ABDALLA DARWISH AND SOTIRIS K. NTOUYAS
}

Received 19 March, 2012

\begin{abstract}
In this paper, we study the existence of solutions for boundary value problems of fractional differential equations of order $q \in(0,1]$ with four-point integral boundary conditions. Existence and uniqueness results are obtained by using well known fixed point theorems. Some illustrative examples are also discussed.
\end{abstract}

2010 Mathematics Subject Classification: 34A08; 34B10; 34B15

Keywords: fractional differential equations, four-point integral boundary conditions, existence, contraction principle, Krasnoselskii's fixed point theorem, Leray-Schauder nonlinear alternative

\section{INTRODUCTION}

In recent years, boundary value problems for nonlinear fractional differential equations have been addressed by several researchers. Fractional derivatives provide an excellent tool for the description of memory and hereditary properties of various materials and processes, see [15]. These characteristics of the fractional derivatives make the fractional-order models more realistic and practical than the classical integer-order models. As a matter of fact, fractional differential equations arise in many engineering and scientific disciplines such as physics, chemistry, biology, economics, control theory, signal and image processing, biophysics, blood flow phenomena, aerodynamics, fitting of experimental data, etc. [13,15-17]. For some recent development on the topic, see [1-11] and the references therein.

In this paper, we discuss the existence and uniqueness of solutions for a boundary value problem of nonlinear fractional differential equations of order $q \in(0,1]$ with four-point integral boundary conditions given by

$$
\left\{\begin{array}{c}
{ }^{c} D^{q} x(t)=f(t, x(t)), 0<t<1,0<q \leq 1, \\
x(0)+\alpha \int_{\mu}^{v} x(s) d s=x(1), 0<\mu<v<1(\mu \neq v),
\end{array}\right.
$$


where ${ }^{c} D^{q}$ denotes the Caputo fractional derivative of order $q, f:[0,1] \times \mathbb{R} \rightarrow \mathbb{R}$ is continuous and $\alpha \in \mathbb{R} \backslash\{0\}$. We denote by $\mathcal{C}=C([0,1], \mathbb{R})$ the Banach space of all continuous functions from $[0,1] \rightarrow \mathbb{R}$ endowed with a topology of uniform convergence with the norm denoted by $\|\cdot\|$.

The boundary condition in the problem (1.1) can be regarded as four-point nonlocal boundary condition, which reduces to the typical integral boundary condition in the limit $\mu \rightarrow 0, v \rightarrow 1$.

We prove some new existence and uniqueness results by using a variety of fixed point theorems. In Theorem 1 we prove an existence and uniqueness result by using Banach's contraction principle, in Theorem 2 we prove the existence of a solution by using Krasnoselskii's fixed point theorem, while in Theorem 3 we prove the existence of a solution via Leray-Schauder nonlinear alternative.

It is worth mention that the methods used in this paper are standard, however their exposition in the framework of problem (1.1) is new.

\section{Preliminaries on Fractional Calculus}

Let us recall some basic definitions of fractional calculus $[13,17]$.

Definition 1. For a continuous function $g:[0, \infty) \rightarrow \mathbb{R}$, the Caputo derivative of fractional order $q$ is defined as

$$
{ }^{c} D^{q} g(t)=\frac{1}{\Gamma(n-q)} \int_{0}^{t}(t-s)^{n-q-1} g^{(n)}(s) d s, n-1<q<n, n=[q]+1,
$$

where $[q]$ denotes the integer part of the real number $q$.

Definition 2. The Riemann-Liouville fractional integral of order $q$ is defined as

$$
I^{q} g(t)=\frac{1}{\Gamma(q)} \int_{0}^{t} \frac{g(s)}{(t-s)^{1-q}} d s, q>0,
$$

provided the integral exists.

Definition 3. The Riemann-Liouville fractional derivative of order $q$ for a continuous function $g(t)$ is defined by

$$
D^{q} g(t)=\frac{1}{\Gamma(n-q)}\left(\frac{d}{d t}\right)^{n} \int_{0}^{t} \frac{g(s)}{(t-s)^{q-n+1}} d s, \quad n=[q]+1,
$$

provided the right hand side is pointwise defined on $(0, \infty)$.

Lemma 1 ([13]). For $q>0$, the general solution of the fractional differential equation ${ }^{c} D^{q} x(t)=0$ is given by

$$
x(t)=c_{0}+c_{1} t+c_{2} t^{2}+\ldots+c_{n-1} t^{n-1},
$$

where $c_{i} \in \mathbb{R}, i=0,1,2, \ldots, n-1(n=[q]+1)$. 
In view of Lemma 1 , it follows that

$$
I^{q c} D^{q} x(t)=x(t)+c_{0}+c_{1} t+c_{2} t^{2}+\ldots+c_{n-1} t^{n-1},
$$

for some $c_{i} \in \mathbb{R}, i=0,1,2, \ldots, n-1(n=[q]+1)$.

Lemma 2. For a given $g \in C([0,1], \mathbb{R})$ the unique solution of the boundary value problem

$$
\left\{\begin{array}{l}
{ }^{c} D^{q} x(t)=g(t), 0<t<1,0<q \leq 1, \\
x(0)+\alpha \int_{\mu}^{v} x(s) d s=x(1), 0<\eta<1
\end{array}\right.
$$

is given by

$$
\begin{aligned}
x(t)= & \frac{1}{\Gamma(q)} \int_{0}^{t}(t-s)^{q-1} g(s) d s+\frac{1}{\alpha(v-\mu) \Gamma(q)} \int_{0}^{1}(1-s)^{q-1} g(s) d s \\
& -\frac{1}{(v-\mu) \Gamma(q)} \int_{\mu}^{v}\left(\int_{0}^{s}(s-m)^{q-1} g(m) d m\right) d s, \quad t \in[0,1] .
\end{aligned}
$$

Proof. For some constant $c_{0} \in \mathbb{R}$, we have

$$
x(t)=I^{q} g(t)-c_{0}=\int_{0}^{t} \frac{(t-s)^{q-1}}{\Gamma(q)} g(s) d s-c_{0} .
$$

We have $x(0)=-c_{0}$,

$$
\begin{aligned}
\alpha \int_{\mu}^{v} x(s) d s & =\alpha \int_{\mu}^{v}\left(\int_{0}^{s} \frac{(s-m)^{q-1}}{\Gamma(q)} g(m) d m-c_{0}\right) d s \\
& =\alpha \int_{\mu}^{v}\left(\int_{0}^{s} \frac{(s-m)^{q-1}}{\Gamma(q)} g(m) d m\right) d s-\alpha c_{0}(v-\mu)
\end{aligned}
$$

and

$$
x(1)=\int_{0}^{1} \frac{(1-s)^{q-1}}{\Gamma(q)} g(s) d s-c_{0},
$$

which imply that

$$
c_{0}=\frac{1}{v-\mu} \int_{\mu}^{v}\left(\int_{0}^{s} \frac{(s-m)^{q-1}}{\Gamma(q)} g(m) d m\right) d s-\frac{1}{\alpha(v-\mu)} \int_{0}^{1} \frac{(1-s)^{q-1}}{\Gamma(q)} g(s) d s .
$$

Substituting the value of $c_{0}$ in (2.3) we obtain the solution (2.2). 


\section{EXISTENCE RESUlTS}

In view of Lemma 2 , we define an operator $\mathbf{F}: \mathcal{\ell} \rightarrow \mathcal{C}$ by

$$
\begin{aligned}
(\mathbf{F} x)(t) & =\frac{1}{\Gamma(q)} \int_{0}^{t}(t-s)^{q-1} f(s, x(s)) d s \\
& +\frac{1}{\alpha(v-\mu) \Gamma(q)} \int_{0}^{1}(1-s)^{q-1} f(s, x(s)) d s \\
& -\frac{1}{(v-\mu) \Gamma(q)} \int_{\mu}^{v}\left(\int_{0}^{s}(s-m)^{q-1} f(m, x(m)) d m\right) d s, \quad t \in[0,1] .
\end{aligned}
$$

In the following we use the norm $\|x\|=\sup _{t \in[0,1]}|x(t)|$ and for convenience, we set

$$
\Lambda=\frac{1}{\Gamma(q+1)}\left(1+\frac{\delta_{1}\left[q+1+|\alpha|\left(v^{q+1}-\mu^{q+1}\right)\right]}{|\alpha|(q+1)}\right), \quad \delta_{1}=\frac{1}{|\nu-\mu|} .
$$

Our first result is based on Banach's contraction principle.

Theorem 1. Assume that $f:[0,1] \times \mathbb{R} \rightarrow \mathbb{R}$ is a continuous function and satisfies the assumption

$\left(A_{1}\right)|f(t, x)-f(t, y)| \leq L|x-y|, \forall t \in[0,1], L>0, x, y \in \mathbb{R}$,

with $L<1 / \Lambda$, where $\Lambda$ is given by (3.2). Then the boundary value problem (1.1) has a unique solution.

Proof. Setting $\sup _{t \in[0,1]}|f(t, 0)|=M$ and choosing $r \geq \frac{\Lambda M}{1-L \Lambda}$, we show that F $B_{r} \subset B_{r}$, where $B_{r}=\{x \in \mathcal{C}:\|x\| \leq r\}$. For $x \in B_{r}$, we have

$$
\begin{aligned}
& \|(\mathbf{F} x)(t)\| \\
& \leq \sup _{t \in[0,1]}\left\{\frac{1}{\Gamma(q)} \int_{0}^{t}(t-s)^{q-1}|f(s, x(s))| d s\right. \\
& +\frac{1}{|\alpha(v-\mu)| \Gamma(q)} \int_{0}^{1}(1-s)^{q-1}|f(s, x(s))| d s \\
& \left.+\frac{1}{|v-\mu| \Gamma(q)} \int_{\mu}^{v}\left(\int_{0}^{s}(s-m)^{q-1}|f(m, x(m))| d m\right) d s\right\} \\
& \leq \sup _{t \in[0,1]}\left\{\frac{1}{\Gamma(q)} \int_{0}^{t}(t-s)^{q-1}(|f(s, x(s))-f(s, 0)|+|f(s, 0)|) \| d s\right. \\
& +\frac{1}{|\alpha(v-\mu)| \Gamma(q)} \int_{0}^{1}(1-s)^{q-1}(|f(s, x(s))-f(s, 0)|+|f(s, 0)|) d s \\
& +\frac{1}{|v-\mu| \Gamma(q)} \int_{\mu}^{v}\left(\int_{0}^{s}(s-m)^{q-1}(|f(m, x(m))-f(m, 0)|\right.
\end{aligned}
$$




$$
\begin{aligned}
& +|f(m, 0)|) d m) d s\} \\
& \leq(L r+M) \sup _{t \in[0,1]}\left\{\frac{1}{\Gamma(q)} \int_{0}^{t}(t-s)^{q-1} d s+\frac{1}{|\alpha(v-\mu)| \Gamma(q)} \int_{0}^{1}(1-s)^{q-1} d s\right. \\
& \left.+\frac{1}{|v-\mu| \Gamma(q)} \int_{\mu}^{v}\left(\int_{0}^{s}(s-m)^{q-1} d m\right) d s\right\} \\
& \leq \frac{(L r+M)}{\Gamma(q+1)}\left(1+\frac{\delta_{1}\left[q+1+|\alpha|\left(v^{q+1}-\mu^{q+1}\right)\right]}{|\alpha|(q+1)}\right) \\
& =(L r+M) \Lambda \leq r .
\end{aligned}
$$

Now, for $x, y \in \mathcal{C}$ and for each $t \in[0,1]$, we obtain

$$
\begin{aligned}
& \|(\mathbf{F} x)(t)-(\mathbf{F} y)(t)\| \\
& \leq \sup _{t \in[0,1]}\left\{\frac{1}{\Gamma(q)} \int_{0}^{t}(t-s)^{q-1}|f(s, x(s))-f(s, y(s))| d s\right. \\
& +\frac{1}{|\alpha(v-\mu)| \Gamma(q)} \int_{0}^{1}(1-s)^{q-1}|f(s, x(s))-f(s, y(s))| d s \\
& \left.+\frac{1}{|v-\mu| \Gamma(q)} \int_{\mu}^{v}\left(\int_{0}^{s}(s-m)^{q-1}|f(m, x(m))-f(m, y(m))| d m\right) d s\right\} \\
& \leq L\|x-y\| \sup _{t \in[0,1]}\left\{\frac{1}{\Gamma(q)} \int_{0}^{t}(t-s)^{q-1} d s+\frac{1}{|\alpha(v-\mu)| \Gamma(q)} \int_{0}^{1}(1-s)^{q-1} d s\right. \\
& \left.+\frac{1}{|v-\mu| \Gamma(q)} \int_{\mu}^{v}\left(\int_{0}^{s}(s-m)^{q-1} d m\right) d s\right\} \\
& \leq \frac{L}{\Gamma(q+1)}\left(1+\frac{\delta_{1}\left[q+1+|\alpha|\left(v^{q+1}-\mu^{q+1}\right)\right]}{|\alpha|(q+1)}\right)\|x-y\|=L \Lambda\|x-y\|,
\end{aligned}
$$

where $\Lambda$ is given by (3.2). Observe that $\Lambda$ depends only on the parameters involved in the problem. As $L<1 / \Lambda$, therefore $\mathbf{F}$ is a contraction. Thus, the conclusion of the theorem follows by the contraction mapping principle (Banach fixed point theorem).

Now, we prove the existence of solution of (1.1) by applying Krasnoselskii's fixed point theorem.

Lemma 3 ([14], Krasnoselskii's fixed point theorem). Let $M$ be a closed, bounded, convex and nonempty subset of a Banach space $X$. Let $A, B$ be the operators such that:

(i) $A x+B y \in M$ whenever $x, y \in M$;

(ii) A is compact and continuous; 
(iii) $B$ is a contraction mapping.

Then there exists $z \in M$ such that $z=A z+B z$.

Theorem 2. Let $f:[0,1] \times \mathbb{R} \rightarrow \mathbb{R}$ be a continuous function satisfying assumption $\left(A_{1}\right)$. Moreover we assume that

$\left(A_{2}\right)|f(t, x)| \leq \mu(t), \quad \forall(t, x) \in[0,1] \times \mathbb{R}$, and $\mu \in C\left([0,1], \mathbb{R}^{+}\right)$.

If

$$
\frac{L}{\Gamma(q+1)}\left(\frac{\delta_{1}\left[q+1+|\alpha|\left(\nu^{q+1}-\mu^{q+1}\right)\right]}{|\alpha|(q+1)}\right)<1,
$$

then the boundary value problem (1.1) has at least one solution on $[0,1]$.

Proof. Letting $\sup _{t \in[0,1]}|\mu(t)|=\|\mu\|$, we fix

$$
\bar{r} \geq \frac{\|\mu\|}{\Gamma(q+1)}\left(1+\frac{\delta_{1}\left[q+1+|\alpha|\left(v^{q+1}-\mu^{q+1}\right)\right]}{|\alpha|(q+1)}\right),
$$

and consider $B_{\bar{r}}=\{x \in \mathcal{C}:\|x\| \leq \bar{r}\}$. We define the operators $\mathcal{P}$ and $\mathcal{Q}$ on $B_{\bar{r}}$ as

$$
\begin{aligned}
(\mathcal{P} x)(t) & =\int_{0}^{t} \frac{(t-s)^{q-1}}{\Gamma(q)} f(s, u(s)) d s, t \in[0,1] \\
(\mathcal{Q} x)(t) & =\frac{1}{\alpha(v-\mu) \Gamma(q)} \int_{0}^{1}(1-s)^{q-1} f(s, x(s) d s \\
& -\frac{1}{(v-\mu) \Gamma(q)} \int_{\mu}^{v}\left(\int_{0}^{s}(s-m)^{q-1} f(m, x(m)) d m\right) d s, t \in[0,1] .
\end{aligned}
$$

For $x, y \in B_{\bar{r}}$, we find that

$$
\|\mathcal{P} x+\mathcal{Q} y\| \leq \frac{\|\mu\|}{\Gamma(q+1)}\left(1+\frac{\delta_{1}\left[q+1+|\alpha|\left(v^{q+1}-\mu^{q+1}\right)\right]}{|\alpha|(q+1)}\right) \leq \bar{r} .
$$

Thus, $\mathcal{P} x+\mathcal{Q} y \in B_{\bar{r}}$. It follows from the assumption $\left(A_{1}\right)$ together with (3.3) that $\mathcal{Q}$ is a contraction mapping. Continuity of $f$ implies that the operator $\mathcal{P}$ is continuous. Also, $\mathcal{P}$ is uniformly bounded on $B_{\bar{r}}$ as

$$
\|\mathcal{P} x\| \leq \frac{\|\mu\|}{\Gamma(q+1)} .
$$

Now we prove the compactness of the operator $\mathcal{P}$.

In view of $\left(A_{1}\right)$, we define $\sup _{(t, x) \in[0,1] \times B_{\bar{r}}}|f(t, x)|=\bar{f}$, and consequently we have

$$
\begin{aligned}
& \left\|(\mathcal{P} x)\left(t_{1}\right)-(\mathcal{P} x)\left(t_{2}\right)\right\| \\
& =\sup _{(t, x) \in[0,1] \times B_{\bar{r}}} \mid \frac{1}{\Gamma(q)} \int_{0}^{t_{1}}\left[\left(t_{2}-s\right)^{q-1}-\left(t_{1}-s\right)^{q-1}\right] f(s, x(s)) d s
\end{aligned}
$$




$$
\begin{aligned}
& +\int_{t_{1}}^{t_{2}}\left(t_{2}-s\right)^{q-1} f(s, x(s)) d s \mid \\
& \leq \frac{\bar{f}}{\Gamma(q+1)}\left|2\left(t_{2}-t_{1}\right)^{q}+t_{1}^{q}-t_{2}^{q}\right|,
\end{aligned}
$$

which is independent of $x$ and tends to zero as $t_{2}-t_{1} \rightarrow 0$. Thus, $\mathcal{P}$ is equicontinuous. Hence, by the Arzelá-Ascoli Theorem, $\mathcal{P}$ is compact on $B_{\bar{r}}$. Thus all the assumptions of Lemma 3 are satisfied. So the conclusion of Lemma 3 implies that the boundary value problem (1.1) has at least one solution on $[0,1]$.

Our next result is based on Leray-Schauder Nonlinear Alternative.

Lemma 4 ([12], Nonlinear alternative for single valued maps). . Let $E$ be a Banach space, $C$ a closed, convex subset of $E, U$ an open subset of $C$ and $0 \in U$. Suppose that $F: \bar{U} \rightarrow C$ is a continuous, compact (that is, $F(\bar{U})$ is a relatively compact subset of $C$ ) map. Then either

(i) $F$ has a fixed point in $\bar{U}$, or

(ii) there is $a u \in \partial U$ (the boundary of $U$ in $C$ ) and $\lambda \in(0,1)$ with $u=\lambda F(u)$.

Theorem 3. Let $f:[0,1] \times \mathbb{R} \rightarrow \mathbb{R}$ be a continuous function. Assume that:

$\left(A_{3}\right)$ There exist a function $p \in L^{1}\left([0,1], \mathbb{R}^{+}\right)$, and $\psi: \mathbb{R}^{+} \rightarrow \mathbb{R}^{+}$nondecreasing such that $|f(t, x)| \leq p(t) \psi(\|x\|), \forall(t, x) \in[0,1] \times \mathbb{R}$.

$\left(A_{4}\right)$ There exists a constant $M>0$ such that

$$
\frac{M}{\frac{\psi(M)}{\Gamma(q)}\left\{\left(1+\frac{\delta_{1}}{|\alpha|}\right) \int_{0}^{1}(1-s)^{q-1} p(s) d s+\delta_{1} \int_{\mu}^{v}\left(\int_{0}^{s}(s-m)^{q-1} p(s) d s\right) d s\right\}}>1,
$$

where $\delta_{1}$ is given by (3.2).

Then the boundary value problem (1.1) has at least one solution on [0,1].

Proof. Consider the operator $F: \mathcal{C} \rightarrow \mathcal{C}$ given by (3.1).

We prove that the operator $F$ is completely continuous. First we prove that $F x$ maps bounded sets into bounded sets in $C([0,1], \mathbb{R})$. Indeed, it is enough to show that there exists a positive constant $\ell$ such that, for each $x \in B_{r}=\{x \in C([0,1], \mathbb{R})$ : $\|x\| \leq r\}$, we have $\|F x\| \leq \ell$. From $\left(A_{3}\right)$ we have

$$
\begin{aligned}
& |(F x)(t)| \\
& =\frac{1}{\Gamma(q)} \int_{0}^{t}(t-s)^{q-1}|f(s, x(s))| d s \\
& +\frac{1}{|\alpha(v-\mu)| \Gamma(q)} \int_{0}^{1}(1-s)^{q-1}|f(s, x(s))| d s \\
& +\frac{1}{|v-\mu| \Gamma(q)} \int_{\mu}^{v}\left(\int_{0}^{s}(s-m)^{q-1}|f(m, x(m))| d m\right) d s
\end{aligned}
$$




$$
\begin{aligned}
& \leq \frac{\psi(\|x\|)}{\Gamma(q)} \int_{0}^{t}(t-s)^{q-1} p(s) d s+\frac{\psi(\|x\|)}{|\alpha(v-\mu)| \Gamma(q)} \int_{0}^{1}(1-s)^{q-1} p(s) d s \\
& +\frac{\psi(\|x\|)}{|v-\mu| \Gamma(q)} \int_{\mu}^{v}\left(\int_{0}^{s}(s-m)^{q-1} p(s) d s\right) d s \\
& \leq \frac{\psi(\|x\|)}{\Gamma(q)}\left\{\left(1+\frac{\delta_{1}}{|\alpha|}\right) \int_{0}^{1}(1-s)^{q-1} p(s) d s\right. \\
& \left.+\delta_{1} \int_{\mu}^{v}\left(\int_{0}^{s}(s-m)^{q-1} p(s) d s\right) d s\right\} .
\end{aligned}
$$

Thus,

$$
\begin{aligned}
\|(F x)\| \leq \frac{\psi(r)}{\Gamma(q)}\left\{\left(1+\frac{\delta_{1}}{|\alpha|}\right) \int_{0}^{1}(1-s)^{q-1} p(s) d s\right. \\
\left.+\delta_{1} \int_{\mu}^{v}\left(\int_{0}^{s}(s-m)^{q-1} p(s) d s\right) d s\right\}:=\ell .
\end{aligned}
$$

Next we show that $F x$ maps bounded sets into equicontinuous sets of $C([0,1], \mathbb{R})$. Let $t^{\prime}, t^{\prime \prime} \in[0,1]$ with $t^{\prime}<t^{\prime \prime}$ and $x \in B_{r}$, where $B_{r}$ is a bounded set of $C([0,1], \mathbb{R})$. Then

$$
\begin{aligned}
\left|(F x)\left(t^{\prime \prime}\right)-(F x)\left(t^{\prime}\right)\right| & =\mid \frac{1}{\Gamma(q)} \int_{0}^{t^{\prime \prime}}\left(t^{\prime \prime}-s\right)^{q-1} f(s, x(s)) d s \\
& -\frac{1}{\Gamma(q)} \int_{0}^{t^{\prime}}\left(t^{\prime}-s\right)^{q-1} f(s, x(s)) d s \mid \\
& \leq\left|\frac{\psi(r)}{\Gamma(q)} \int_{0}^{t^{\prime}}\left[\left(t^{\prime \prime}-s\right)^{q-1}-\left(t^{\prime}-s\right)^{q-1}\right] p(s) d s\right| \\
& +\left|\frac{\psi(r)}{\Gamma(q)} \int_{t^{\prime}}^{t^{\prime \prime}}\left(t^{\prime \prime}-s\right)^{q-1} p(s) d s\right| .
\end{aligned}
$$

Obviously the right hand side of the above inequality tends to zero independently of $x \in B_{r}$ as $t^{\prime \prime}-t^{\prime} \rightarrow 0$. Therefore it follows by the Arzelá-Ascoli theorem that $F: C([0,1], \mathbb{R}) \rightarrow C([0,1], \mathbb{R})$ is completely continuous.

Now let $\lambda \in(0,1)$ and let $x=\lambda F x$. Then for $x \in[0,1]$, using the previous computations in proving that $F x$ is bounded, we have

$$
\begin{aligned}
|x(t)| & =|\lambda(F x)(t)| \\
& \leq \frac{1}{\Gamma(q)} \int_{0}^{t}(t-s)^{q-1}|f(s, x(s))| d s \\
& +\frac{1}{|\alpha(v-\mu)| \Gamma(q)} \int_{0}^{1}(1-s)^{q-1}|f(s, x(s))| d s
\end{aligned}
$$




$$
\begin{gathered}
+\frac{1}{|v-\mu| \Gamma(q)} \int_{\mu}^{v}\left(\int_{0}^{s}(s-m)^{q-1}|f(m, x(m))| d m\right) d s \\
\frac{\psi(\|x\|)}{\Gamma(q)}\left\{\left(1+\frac{\delta_{1}}{|\alpha|}\right) \int_{0}^{1}(1-s)^{q-1} p(s) d s\right. \\
\left.+\delta_{1} \int_{\mu}^{v}\left(\int_{0}^{s}(s-m)^{q-1} p(s) d s\right) d s\right\} .
\end{gathered}
$$

and consequently

$$
\frac{\|x\|}{\frac{\psi(\|x\|)}{\Gamma(q)}\left\{\left(1+\frac{\delta_{1}}{|\alpha|}\right) \int_{0}^{1}(1-s)^{q-1} p(s) d s+\delta_{1} \int_{\mu}^{v}\left(\int_{0}^{s}(s-m)^{q-1} p(s) d s\right) d s\right\}} \leq 1 .
$$

In view of $\left(A_{4}\right)$, there exists $M$ such that $\|x\| \neq M$. Let us set

$$
U=\{x \in C([0,1], X):\|x\|<M\} .
$$

Note that the operator $F: \bar{U} \rightarrow C([0,1], \mathbb{R})$ is continuous and completely continuous. From the choice of $U$, there is no $x \in \partial U$ such that $x=\lambda F(x)$ for some $\lambda \in(0,1)$. Consequently, by the nonlinear alternative of Leray-Schauder type (Lemma 4$)$, we deduce that $F$ has a fixed point $x \in \bar{U}$ which is a solution of the problem (1.1). This completes the proof.

In the special case when $p(t)=1$ and $\psi(|x|)=k|x|+N$ we have the following corollary.

Corollary 1. Let $f:[0,1] \times \mathbb{R} \rightarrow \mathbb{R}$. Assume that:

$\left(A_{5}\right)$ there exist constants $0 \leq \kappa<\frac{1}{\Lambda}$, where $\Lambda$ is given by (3.2) and $M>0$ such that

$$
|f(t, x)| \leq \kappa|x|+M, \quad \text { for all } \quad t \in[0,1], x \in C[0,1] .
$$

Then the boundary value problem (1.1) has at least one solution.

\section{EXAMPLES}

Example 1. Consider the following four-point integral fractional boundary value problem

$$
\left\{\begin{array}{c}
{ }^{c} D^{1 / 2} x(t)=\frac{1}{(t+9)^{2}} \frac{|x|}{1+|x|}, \quad t \in[0,1], \\
x(0)+\int_{1 / 4}^{3 / 4} x(s) d s=x(1) .
\end{array}\right.
$$


Here, $q=1 / 2, \alpha=1, \mu=1 / 4, v=3 / 4$, and $f(t, x)=\frac{1}{(t+9)^{2}} \frac{|x|}{1+|x|}$. As $|f(t, x)-f(t, y)| \leq \frac{1}{81}|x-y|$, therefore, $\left(A_{1}\right)$ is satisfied with $L=\frac{1}{81}$. Further,

$$
L \Lambda=\frac{L}{\Gamma(q+1)}\left(1+\frac{\delta_{1}\left[q+1+|\alpha|\left(v^{q+1}-\mu^{q+1}\right)\right]}{|\alpha|(q+1)}\right)=\frac{17+3 \sqrt{3}}{729 \sqrt{\pi}}<1 .
$$

Thus, by the conclusion of Theorem 1, the boundary value problem (4.1) has a unique solution on $[0,1]$.

Example 2. We consider the following boundary value problem

$$
\left\{\begin{array}{l}
{ }^{c} D^{1 / 2} x(t)=\frac{1}{(16 \pi)} \sin (2 \pi x)+\frac{|x|}{1+|x|}, t \in[0,1], \\
x(0)+\int_{1 / 4}^{3 / 4} x(s) d s=x(1) .
\end{array}\right.
$$

Here, $q=1 / 2, \alpha=1, \mu=1 / 4, v=3 / 4$, and

$$
|f(t, x)|=\left|\frac{1}{(16 \pi)} \sin (2 \pi x)+\frac{|x|}{1+|x|}\right| \leq \frac{1}{8}|x|+1 .
$$

Clearly $M=1$ and

$$
\kappa=\frac{1}{8}<\frac{1}{\Lambda}=\frac{9 \sqrt{\pi}}{17+3 \sqrt{3}} .
$$

Thus, all the conditions of Corollary 1 are satisfied and consequently the problem (4.2) has at least one solution.

\section{REFERENCES}

[1] B. Ahmad, "Existence of solutions for fractional differential equations of order $q \in(2,3]$ with anti-periodic boundary conditions," J. Appl. Math. Comput., vol. 34, no. 1-2, pp. 385-391, 2010.

[2] B. Ahmad, "Existence of solutions for irregular boundary value problems of nonlinear fractional differential equations," Appl. Math. Lett., vol. 23, no. 4, pp. 390-394, 2010.

[3] B. Ahmad and R. P. Agarwal, "On nonlocal fractional boundary value problems," Dyn. Contin. Discrete Impuls. Syst., Ser. A, Math. Anal., vol. 18, no. 4, pp. 535-544, 2011.

[4] B. Ahmad and A. Alsaedi, "Existence and uniqueness of solutions for coupled systems of higherorder nonlinear fractional differential equations," Fixed Point Theory Appl., vol. 2010, p. 17, 2010.

[5] B. Ahmad and J. J. Nieto, "Existence of solutions for nonlocal boundary value problems of higherorder nonlinear fractional differential equations," Abstr. Appl. Anal., vol. 2009, p. 9, 2009.

[6] B. Ahmad and J. J. Nieto, "Existence results for a coupled system of nonlinear fractional differential equations with three-point boundary conditions," Comput. Math. Appl., vol. 58, no. 9, pp. 1838-1843, 2009.

[7] B. Ahmad and J. J. Nieto, "Existence results for nonlinear boundary value problems of fractional integrodifferential equations with integral boundary conditions," Bound. Value Probl., vol. 2009, p. 11, 2009. 
[8] B. Ahmad and S. Sivasundaram, "On four-point nonlocal boundary value problems of nonlinear integro-differential equations of fractional order," Appl. Math. Comput., vol. 217, no. 2, pp. 480 $487,2010$.

[9] Z. Bai, "On positive solutions of a nonlocal fractional boundary value problem," Nonlinear Anal., Theory Methods Appl., Ser. A, Theory Methods, vol. 72, no. 2, pp. 916-924, 2010.

[10] K. Balachandran and J. J. Trujillo, "The nonlocal Cauchy problem for nonlinear fractional integrodifferential equations in Banach spaces," Nonlinear Anal., Theory Methods Appl., Ser. A, Theory Methods, vol. 72, no. 12, pp. 4587-4593, 2010.

[11] M. Benchohra, S. Hamani, and S. Ntouyas, "Boundary value problems for differential equations with fractional order and nonlocal conditions," Nonlinear Anal., Theory Methods Appl., Ser. A, Theory Methods, vol. 71, no. 7-8, pp. 2391-2396, 2009.

[12] A. Granas and J. Dugundji, Fixed point theory, ser. Springer Monographs in Mathematics. New York, NY: Springer, 2003.

[13] A. A. Kilbas, H. M. Srivastava, and J. J. Trujillo, Theory and applications of fractional differential equations., ser. North-Holland Mathematics Studies. Amsterdam: Elsevier, 2006, vol. 204.

[14] M. A. Krasnoselskii, "Two remarks on the method of successive approximations," Uspekhi Mat. Nauk, vol. 10, pp. 123-127, 1955.

[15] I. Podlubny, Fractional differential equations. An introduction to fractional derivatives, fractional differential equations, to methods of their solution and some of their applications, ser. Mathematics in Science and Engineering. San Diego, CA: Academic Press, 1999, vol. 198.

[16] J. Sabatier, O. P. Agrawal, and J. A. T. Machado, Eds., Advances in Fractional Calculus, ser. Theoretical Developments and Applications in Physics and Engineering. Dordrecht: Springer, 2007.

[17] S. G. Samko, A. A. Kilbas, and O. I. Marichev, Fractional integrals and derivatives: theory and applications. Transl. from the Russian. New York, NY: Gordon and Breach, 1993.

\section{Authors' addresses}

Mohamed Abdalla Darwish

Department of Mathematics, Sciences Faculty for Girls, King Abdulaziz University, Jeddah, Saudi Arabia, and Department of Mathematics, Faculty of Science, Damanhour University, Damanhour, Egypt

E-mail address: dr.madarwishegmail.com

\section{Sotiris K. Ntouyas}

Department of Mathematics, University of Ioannina, 45110 Ioannina, Greece

E-mail address: sntouyas@uoi.gr 